\title{
Robust Biometric System Using Palmprint for Personal Verification
}

\author{
G.S. Badrinath and Phalguni Gupta \\ Dept. of Computer Science and Engineering, Indain Institute of Technology Kanpur, India \\ \{badri,pg\}@itk.ac.in
}

\begin{abstract}
This paper describes a prototype of robust biometric system for verification. The system uses features extracted using Speeded Up Robust Features (SURF) operator of human hand. The hand image for features is acquired using a low cost scanner. The extracted palmprint region is robust to hand translation and rotation on the scanner. The system is tested on IITK database and PolyU database. It has FAR $0.02 \%$, FRR $0.01 \%$ and an accuracy of $99.98 \%$ at original size. The system addresses the robustness in the context of scale, rotation and occlusion of palmprint. The system performs at accuracy more than $99 \%$ for scale, more than $98 \%$ for rotation, and more than $99 \%$ for occlusion. The robustness and accuracy suggest that it can be a suitable system for civilian and high-security environments.
\end{abstract}

Keywords: Robust, Scale, Occlusion, Rotation, Translation, Scanner.

\section{Introduction}

Biometrics establishes identity of a person by physiological and/or behavioral characteristics. Identification / verification systems based on biometrics have found its applications widely in commercial and law enforcement applications. Systems based on fingerprint features are most widely used and biometric features generated from iris are considered to be the most reliable biometric features [22]. Using human hand as biometric feature is relatively a new approach. Features like principle lines, wrinkles, ridges, minutiae points, singular points, and texture pattern in the palmprint region between wrist and fingers can be used for its representation [20]. Furthermore, systems based on hand features are the most acceptable to users [8].

Limited work has been reported on palmprint identification and verification, despite the importance of palmprint features. Some of the recent research efforts in [1-3], [11] address the problem of palmprint recognition in large databases, and achieve very low error rates. There exist verification/identication system based on palmprint extracted features using various transforms such as Fourier Transform [10], Discrete Cosine Transform [3], Karhunen-Loeve transform [1], Wavelet transform [2],[4],[11] Fisher Discriminant Analysis [16], Gabor filtering [2], Neural networks [3,7] and Independent component Analysis [15]. Also there are systems based on datam points [6], local binary pattern histograms [5], statistical signatures [4], hand geometry features [3]. Furthermore, there exist multimodal systems fusing feature from 
hand-geometry and face [12], eigenpalm and eigenfinger [1] and fusing palmprint features extracted using haar wavelet and principle component anlysis [11].

In [6], [7], systems using ink marking to capture the palmprint patterns have been presented. The systems are not widely accepted due to considerable attention and high cooperation is required in providing a biometric sample. In recent papers on palmprint based recognition system, palmprint images are captured from a digital camera [2] and users hand is placed in constrained environment using pegs for palmprint image acquisition. The problem with peg based system is that the user may not able to enroll or verify themselves if not able to place his/her hand in between the pegs due to injuries or physically challenged. Hence, there is a need to build a system which is

- Robust to translation and rotation of hand image on the scanner.

- Robust to spatial resolution (scale) of the image.

- Robust to occlusion (partially exposed) of the hand image.

- Robust to rotation of the palmprint image given for verification.

Further, it should be highly accurate, available at reasonable cost so that it is suitable for civilian and high end security applications.

This paper has proposed a novel method to extract features in palmprint which can be used in personal verification system. The proposed method tries to incorporate some of the above said characteristics in the palmprint based biometric system. It makes use of Speeded Up Robust Features (SURF) [14] to detect key-points in a given palmprint and determines local features around the key-points as its descriptors. Number of matching key-points for verification is carried out using nearest neighbour ratio method [21]. The hand images are acquired using low cost flat bed scanner.

\section{Speeded Up Robust Features (SURF)}

The SURF [14] is recently emerged cutting edge methodology for pattern recognition, and has been used in general object recognition and for other machine vision applications [17], [18]. SURF has been designed for extracting highly distinctive invariant features from images. The following major stages are used to compute the SURF feature vectors of a given image.

\subsection{Key-Point Detectors}

The SURF key-point detector is based on hessian matrix. Given a point $P=(x, y)$ in an image $I$, the hessian matrix $H=(x, \sigma)$ in $P$ at scale $\sigma$ is defined as follows

$$
H(P, \sigma)=\left[\begin{array}{ll}
L_{x x}(P, \sigma) & L_{x y}(P, \sigma) \\
L_{x y}(P, \sigma) & L_{y y}(P, \sigma)
\end{array}\right]
$$

where $L_{x x}(P, \sigma), L_{x y}(P, \sigma)$ and $L_{y y}(P, \sigma)$ are the convolution of the Gaussian second order derivatives $\frac{\partial^{2}}{\partial x^{2}} g(\sigma), \frac{\partial^{2}}{\partial x \partial y} g(\sigma)$ and $\frac{\partial^{2}}{\partial y^{2}} g(\sigma)$ with the image $I$ at the point $P$ respectively. The second order Gaussian derivatives are approximated using box filters. Image convolutions with box filters are computed rapidly using integral images [19]. Key-points are localized in scale and image space by applying a non maximum suppression in a $3 \times 3 \times 3$ neighborhood. 


\subsection{Key-Point Descriptor}

This stage consists of two steps. In first step, a circular region is constructed around the extracted key-points. Dominant orientation of the circular region is computed using the Haar wavelets response in both $x$ and $y$ directions. The resulting maximum is considered as dominant orientation and used to generate the key-point feature vector. Since, the feature vector of the key-point are measured relative to the dominant orientation, hence the generated feature vectors are invariance to image rotation.

In second step a square region is constructed around each extracted key-point and aligned along the dominant orientation. The square region is partitioned into sixteen smaller sub-regions of size $4 \times 4$. Haar wavelet responses are computed for each subregion. The sum of the wavelet responses $d_{x}$ and $d_{y}$ for each sub-region are used as feature values. Furthermore, the absolute values $\left|d_{x}\right|$ and $|d y|$ are summed to obtain the polarity of the image intensity changes. Thus, the feature vector $V e c$ of the sub-image is given by

$$
V e c=\left\{\sum d_{x}, \sum d_{x}, \sum\left|d_{x}\right|, \sum\left|d_{y}\right|\right\}
$$

The SURF feature vector of the key-point is formed concatenating the feature vectors $V e c$ from all sixteen $4 \times 4$ sub-regions around key-point. So the feature vector of keypoint contains $16 \times 4=64$ elements as the SURF feature vector.

\section{Proposed System}

This section presents a robust biometric system for verification using SURF features of palmprint. Like other biometric systems, it consists of four major stages, described in the following sections.

\subsection{Image Acquisition}

Hand images are obtained at a spatial resolution of 200 dots per inch in gray scale using a flatbed scanner. The device is constraints (pegs) free. Typical gray level image obtained from the scanner is shown in Fig. 1a.

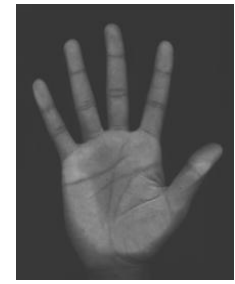

(a)

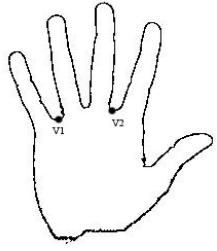

(b)

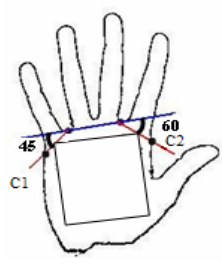

(c)

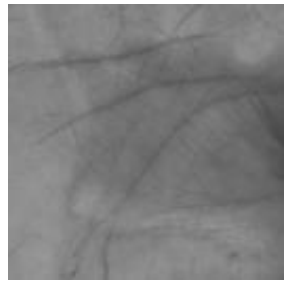

(d)

Fig. 1. (a) Scanned Image, (b) Hand Contour and reference point, (c) Relevant points and Region of interest (palmprint), (d) Extracted Region of Interest (palmprint) 


\subsection{Pre-processing and Region of Interest Extraction}

In this phase hand image is pre-processed and palmprint region is extracted. Global thresholding has been applied to extract the hand from the background. The contour of the hand image as shown in Fig. 1b is extracted applying contour-tracing algorithm [13] on the binarised image. Two reference points $(V I, V 2)$ between the fingertips and the valleys are determined on the contour of hand image as shown in Fig $1 \mathrm{~b}$.

Square area as shown in Fig. 1c with two of its corners placed on middle of line segments $C 1-V 1$ and $V 2-C 2$ is considered as region of interest or palmprint. The line segments $C 1-V 1$ and $V 2-C 2$ are inclined at an angle of $45^{\circ}$ and $60^{\circ}$ respectively to the line joining $V 1$ and $V 2$. The extracted region of interest of the original gray scale image is shown in Fig. 1d.
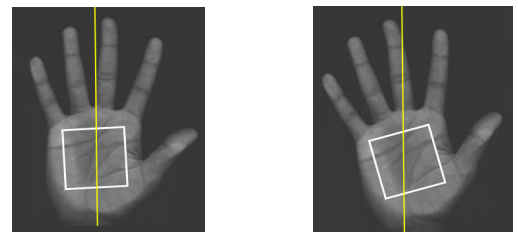

(a)
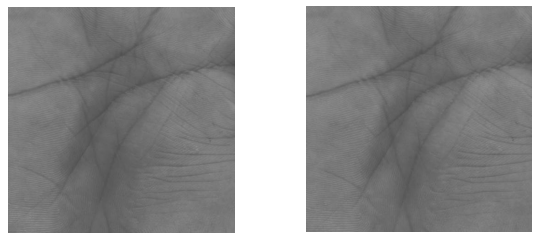

(b)

Fig. 2. (a) Images of same subject with different orientation of placement relative to the symmetry (Yellow Line) of work surface, (b) Extracted region of interest for images shown in Fig. 5a

Since placement of palm on the scanner is pegs free, orientation of placement would vary for every incident. Two images of same subject with different orientations of placement are shown in Fig. 2a. The extracted region of interest or palmprint is relative to the reference points $V 1$ and $V 2$ which are stable for the subject. So the extracted palmprint region remains the same as shown in Fig. $2 b$ for the subject independent to the orientation of placement. Hence the proposed extraction procedure of the system for extracting palmprint makes the system robust to rotation. From the experiments it is observed that the system is robust to rotation for about $\pm 35^{0}$.

\subsection{Palmprint Feature Extraction}

After pre-processing of hand image, and region of interest or palmprint extraction, feature are extracted for verification. SURF is used to extract the features which provides good discrimination ability. The SURF extracted features of the image are

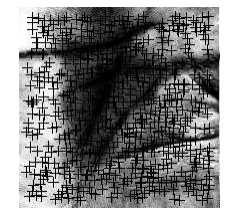

Fig. 3. Detected SURF key-points of the Palmprint 
found to be invariant to scaling, rotation, and translation of the image [14]. Thus extracted feature from an image can be matched correctly with high probability against features from a large image database. The detected SURF key-points for the palmprint image is shown in Fig. 3.

\subsection{Matching}

To verify the live palmprint, the SURF features computed for the enrolled image should be matched with SURF features of the live palmprint image. Matching between SURF key-points of live and enrolled palmprint image is done using nearest neighbor ratio matching [21]. Based on the number of matching points between the live image and enrolled image, a decision is made. Thus more the number of matching points between two images, greater is the similarity between them. To illustrate the matching of extracted palmprint using SURF operator, an example is shown in Fig 4a. Matching between palmprint images of different users is called as imposter matching. An example of imposter matching between two users in IITK database is shown in Fig 4b.

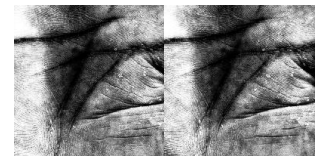

(i) Extracted palmprint ( Id: 87_00 and 87_01) as input pair

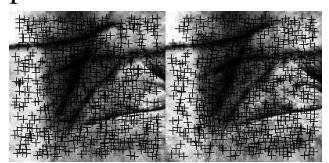

(ii) 627 and 607 Key-points detected for the input pair

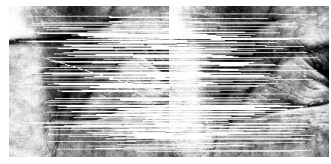

(iii) 210 SURF Matching Key-points obtained for the input pair

(a)

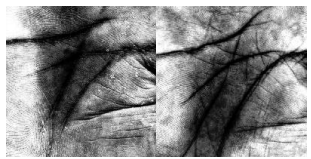

(i) Extracted palmprint (Id: 87_00, and 21_01) as input pair

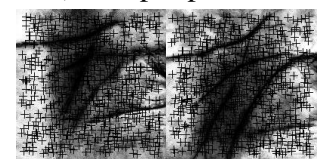

(ii) 627 and 656 Key-points detected for the input pair

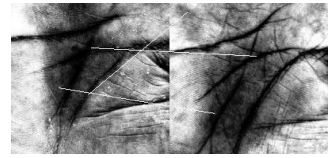

(iii) 3 SURF Matching key-points obtained for the input pair

(b)

Fig. 4. (a) Illustration of genuine palmprint matching using SURF operator, (b) Illustration of imposter matching using SURF operator

\section{Experimental Results}

The proposed system is tested on two sets of image databases. (1) The Indian Institute of Technology Kanpur (IITK) database, and (2) The Hong Kong Polytechnic University (PolyU) database. 


\subsection{Datasets}

IITK has collected a database comprised of 200 images from 100 subjects. All hand images are collected at a spatial resolution of 200 dots per inch using a low cost flat bed scanner, and 256 gray levels. The database is divided into training set and testing set. One image per subject is considered for training, while other image of the subject is used for testing.

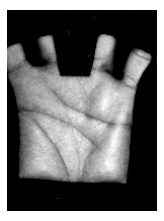

(a)

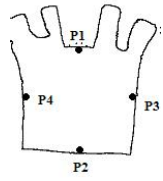

(b)

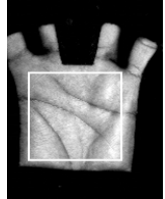

(c)

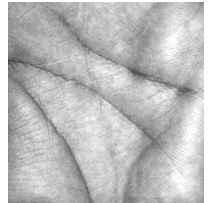

(d)

Fig. 5. (a) PolyU sample image, (b) Reference points, (c) Region of interest in gray scale image, (d) Extracted palmprint

The proposed system has also been tested on the database from PolyU [9]. The images are collected at spatial resolution of 75 dots per inch, and 256 gray levels using CCD [2]. Images are captured placing pegs. Around 17 images per palm are collected in two sessions. 3200 grayscale images corresponding to 200 different palms are used for the experiment. Fig. 5a shows the sample of database. The database is divided into testing set and training set. Four images per palm are considered for training, while remaining twelve images of the palm is considered for testing.

Table 1. Accuracy, FAR and FRR of the proposed system and [11]

\begin{tabular}{||l|c|c|c|c|c|c||}
\hline \multirow{2}{*}{} & \multicolumn{3}{|c|}{ ITT database } & \multicolumn{3}{c||}{ PolyU Database } \\
\cline { 2 - 7 } & FAR & FRR & Accuracy & FAR & FRR & Accuracy \\
\hline Eigenpalm [11] & 9.65 & 9.43 & 90.45 & 6.44 & 8.38 & 92.58 \\
\hline Haarpalm [11] & 5.00 & 13.8 & 90.57 & 4.56 & 12.8 & 91.28 \\
\hline Fusion [11] & 4.73 & 5.66 & 94.80 & 4.17 & 8.10 & 93.85 \\
\hline Proposed & $\mathbf{0 . 0 2}$ & $\mathbf{0 . 0}$ & $\mathbf{9 9 . 9 8}$ & $\mathbf{0 . 0}$ & $\mathbf{0 . 0}$ & $\mathbf{1 0 0 . 0}$ \\
\hline
\end{tabular}

In order to extract region of interest for PolyU database following method is proposed. Four reference points $P 1, P 2, P 3$ and $P 4$ are located on the contour of palm as shown in Fig. 5b. In gray-scale image extract 200 x 200 pixels palm area with its center coinciding with intersecting point of line segments $P 1-P 2$ and $P 3-P 4$. Fig. 5c shows the region of interest in gray scale image, and the extracted palmprint image is shown in Fig. 5d. 


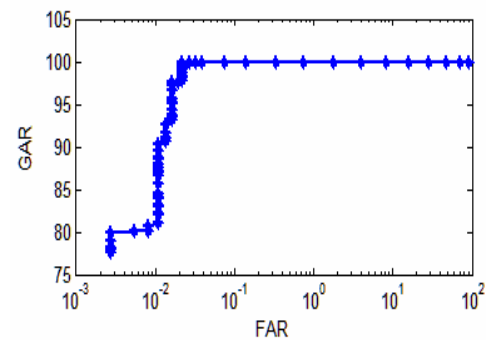

(a)

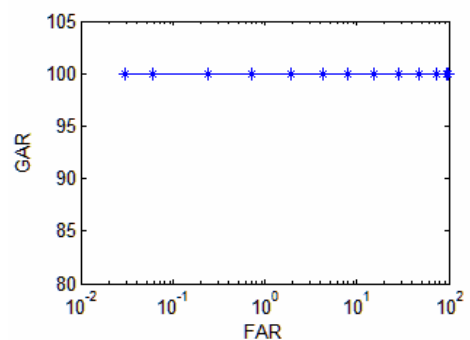

(b)

Fig. 6. (a) ROC curve for IITK database, (b) ROC curve for PolyU database

The experiment has been performed for both the datasets using SURF. The Receiver Operating Characteristic (ROC) curves of the proposed system for is shown in Fig. 6a and Fig. 6b respectively, The proposed system has been compared with known available system [11]. Table 1 shows the accuracy, FAR and FRR of the proposed and previous [11] systems for both the datasets.

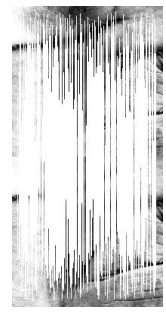

(a) $1.0 \mathrm{~W} \mathrm{x}$ $1.0 \mathrm{H}$

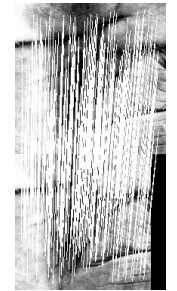

(b) $0.9 W \mathrm{x}$ $0.9 H$

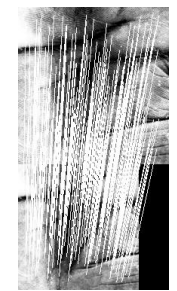

(c) $0.8 \mathrm{~W} \mathrm{x}$ $0.8 H$

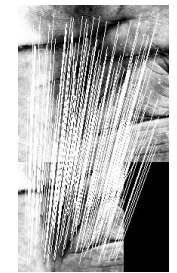

(d) $0.7 \mathrm{Wx}$ $0.7 H$

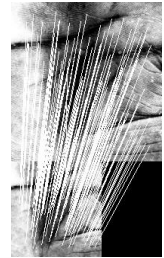

(e) $0.6 \mathrm{~W} \mathrm{x}$ $0.6 H$

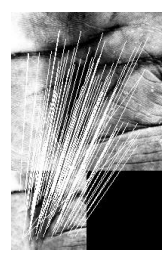

(f) $0.5 W \mathrm{x}$ $0.5 H$

Fig. 7. Surf matching key-points between enrolled image with downscaled palmprint image

\subsection{Scale Invariant}

Since key-point features which are extracted using SURF approach is invariant to scale of the image, the system is robust to be spatial resolution. In order to investigate the robustness to scale, palmprint images in the testing set are down scaled using bicubic interpolation. The testing set of palmprint images are scaled to $0.9 \mathrm{~W} \times 0.9 \mathrm{H}$, $0.8 W \times 0.8 H, 0.7 W \times 0.7 H, 0.6 W \times 0.6 H$, and $0.5 W \times 0.5 H$ to test the system, where $W$ and $H$ are the width and height of the enrolled images respectively. Matching points between the enrolled image and scaled testing palmprint images of the same subject are shown in Fig. 7. The ROC curve for matching with different scales of test images on different datasets are shown in Fig. 8a and Fig. 8b respectively. 


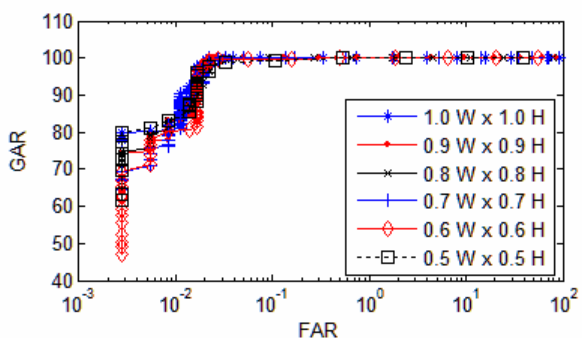

(a)

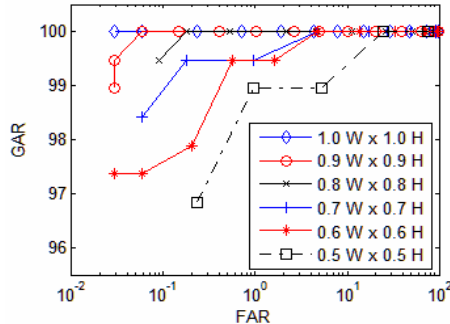

(b)

Fig. 8. (a) ROC curve for IITK database on different scales of the test images, (b) ROC curve for PolyU database on different scales of the test images

Table 2. Accuracy obtained at different scales of testing image

\begin{tabular}{||c|c|c|c|c|c|c||}
\hline & $1.0 \mathrm{~W} \times 1.0 \mathrm{H}$ & $0.9 \mathrm{~W} \times 0.9 \mathrm{H}$ & $0.8 \mathrm{~W} \times 0.8 \mathrm{H}$ & $0.7 \mathrm{~W} \times 0.7 \mathrm{H}$ & $0.6 \mathrm{~W} \times 0.6 \mathrm{H}$ & $0.5 \mathrm{~W} \times 0.5 \mathrm{H}$ \\
\hline $\mathrm{IITK}$ & $99.98 \%$ & $99.98 \%$ & $99.95 \%$ & $99.92 \%$ & $99.83 \%$ & $99.74 \%$ \\
\hline PolyU & $100.0 \%$ & $99.97 \%$ & $99.91 \%$ & $99.65 \%$ & $99.46 \%$ & $98.99 \%$ \\
\hline
\end{tabular}

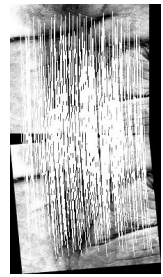

(a) $5^{0}$

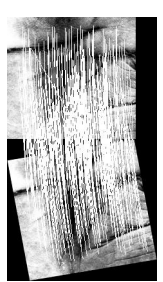

(b) $10^{0}$

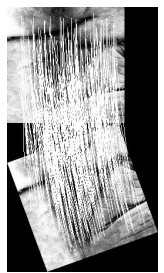

(c) $20^{\circ}$

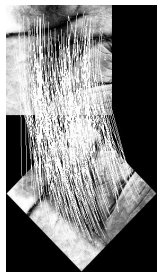

(d) $45^{0}$

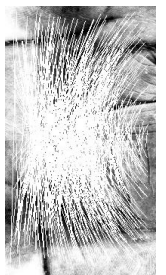

(e) $90^{\circ}$

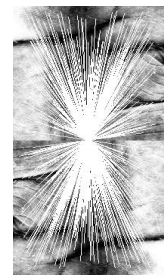

(f) $180^{\circ}$

Fig. 9. Surf matching key-points between enrolled image and rotated palmprint image

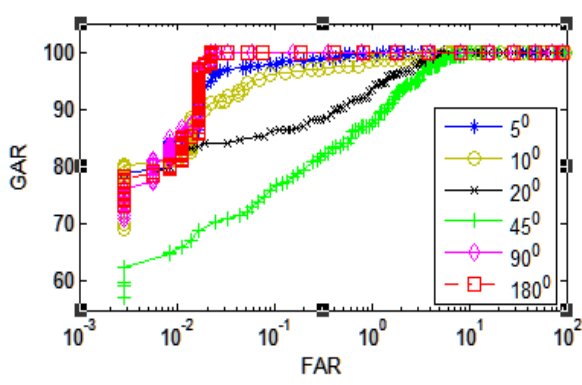

(a)

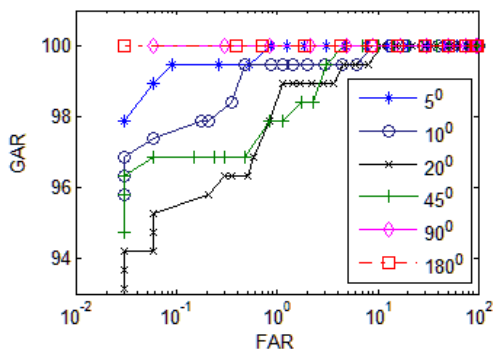

(b)

Fig. 10. (a) ROC curve for IITK database on different rotation of the test images, (b) ROC curve for PolyU database on different rotation of the test images 
The accuracy obtained for the different scales of testing images on IITK and PolyU database is shown in Table 2. It is observed that the accuracy of the system came down below $99 \%$ for scale of the testing images less than $0.5 \mathrm{~W} \times 0.5 \mathrm{H}$ of enrolled image size. From Table 2, it can be infered that the proposed system is highly robust to scale.

\subsection{Rotation Invariant}

The SURF feature vectors of a key-point are relative to the dominant orientation of the key-point. Hence the key-point featuress remain the same irrespective to the orientation of the palmprint image. So the proposed system is robust to the rotation of palmprint image.

Table 3. Accuracy obtained at different rotation of testing image

\begin{tabular}{||c|c|c|c|c|c|c||}
\hline & $5^{0}$ & $10^{0}$ & $20^{0}$ & $45^{0}$ & $90^{0}$ & $180^{0}$ \\
\hline IITK & $99.59 \%$ & $98.82 \%$ & $98.52 \%$ & 98.08 & 99.98 & 99.98 \\
\hline PolyU & $99.69 \%$ & $99.50 \%$ & $98.92 \%$ & $98.54 \%$ & $100.0 \%$ & $100.0 \%$ \\
\hline \hline
\end{tabular}

In order to investigate the robustness to rotation, palmprint images are rotated synthetically using bi-cubic interpolation. The palmprint images in the testing set rotated by $5^{\circ}, 10^{\circ}, 20^{\circ}, 45^{\circ}, 90^{\circ}$, and $180^{\circ}$ are used to test the system. Matching points between the enrolled palmprint image of a subject with different orientation of testing palmprint image are shown in Fig. 9. ROC curve for IITK and PolyU database at different orientations of test images is shown in Fig. 10a and Fig. 10b. The accuracy for different orientation of test image on IITK and PolyU is shown in Table 3. From the table it can be seen that the accuracy of the proposed system is above $98 \%$ independent to rotation of the testing image, which implies the system is highly robust to rotation.

\subsection{Occlusion Invariant}

The proposed system extracts features using SURF which describes the image with local region around key-points. Hence they are independent to area of occlusion. So the proposed system can work in the places where user is able to expose partial palm region to the scanner and remaining region occluded.

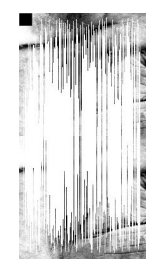

(a) $0.1 \mathrm{~W} \times 0.1 \mathrm{H}$

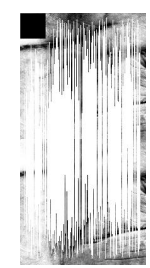

(b) $0.2 \mathrm{~W} \times 0.2 \mathrm{H}$

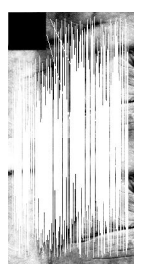

(c) $0.3 \mathrm{~W} \times 0.3 H$
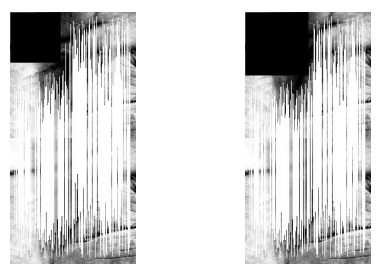

(d) $0.4 \mathrm{~W} \times 0.4 H$

(e) $0.5 \mathrm{~W} \times 0.5 H$

Fig. 11. Surf matching key-points between enrolled image and occluded palmprint image 


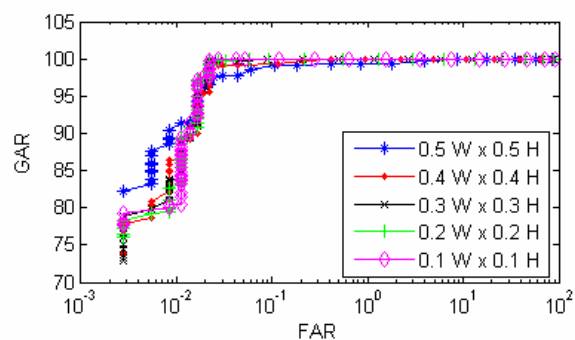

(a)

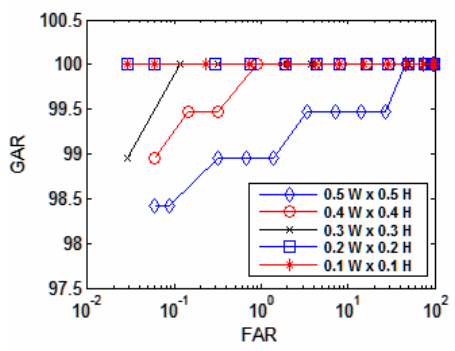

(b)

Fig. 12. (a) ROC curve on IITK database for occlusion of the test images, (b) ROC curve for occlusion of the test images on PolyU database

In order to investigate the robustness to occlusion, palmprint images in the testing set are synthetically occluded with black square region. The typical palmprint images used to test the system are occluded with $0.1 W \times 0.1 H, 0.2 W \times 0.2 H, 0.3 W \times 0.3 H$, $0.4 \mathrm{~W} \times 0.4 \mathrm{H}$, and $0.5 \mathrm{~W} \times 0.5 \mathrm{H}$ of the image. Fig. 11 shows an example for matching key-points between the enrolled palmprint image and occluded testing image of the same subject. ROC curves for both the datasets at different size of occlusions are shown in Fig. 12a and Fig. 12b respectively. The accuracy obtained at different sizes of occlusion on testing images is shown in Table 4. From the results in Table IV it can be inferred that the proposed system at occlusion of $0.5 \mathrm{~W} \times 0.5 \mathrm{H}$ performs with accuracy more than $99 \%$. Hence it implies the proposed system is highly robust to occlusion.

Table 4. Accuracy obtained for different size of occlusion in the testing image

\begin{tabular}{||c|c|c|c|c|c||}
\hline \hline & $0.1 \mathrm{~W} \times 0.1 \mathrm{H}$ & $0.2 \mathrm{~W} \times 0.2 \mathrm{H}$ & $0.3 \mathrm{~W} \times 0.3 \mathrm{H}$ & $0.4 \mathrm{~W} \times 0.4 \mathrm{H}$ & $0.5 \mathrm{~W} \times 0.5 \mathrm{H}$ \\
\hline IITK & $99.98 \%$ & $99.97 \%$ & $99.96 \%$ & $99.80 \%$ & $99.48 \%$ \\
\hline PolyU & $100.0 \%$ & $100.0 \%$ & $99.94 \%$ & $99.66 \%$ & $99.31 \%$ \\
\hline \hline
\end{tabular}

From results in Table 1, it can be inferred that the proposed system performs better than the recent best known system [11] which fuses the matching scores of the haar wavelet and eigenpalm classifiers. The proposed system also addresses the robustness in context of scale, rotation and occlusion of palmprint, and its performance is shown in Table 2, 3, and 4.

\section{Conclusions}

In this paper the use of SURF features in the context of palmprint verification has been investigated. A technique to extract palmprint from hand-image image which is robust to rotation, translation on the scanner surface has been presented. The extracted palmprint is found to be invariant to orientation and translation of palm on scanner, which makes the system robust to rotation and translation. The proposed system is tested on IITK and PolyU databases. The proposed key-point descriptors extracted 
using SURF outperform the earlier known system which fuses the matching scores of the haar wavelet and eigenpalm classifiers [11]. The recognition accuracy of the system is $99.98 \%$, along with FAR $0.021 \%$ and FRR $0.01 \%$ at original sizes. The system is found to be robust to scale, rotation and occlusion. Thus the design of the system with robustness, performance and use of low cost scanner for acquisition of palm image has demonstrated the possibility of using this system for high-end security, and civilian applications.

\section{References}

1. Ribaric, S., Fratric, I.: A biometric identification system based on Eigenpalm and Eigenfinger features. IEEE Trans. on Pattern Analysis Machine Intelligence 27(11), 1698-1709 (2005)

2. Zhang, D., Kong, W.K., You, J., Wong, M.: Online palmprint identification. IEEE Transaction on Pattern Analysis and Machine Intelligence 25(9), 1041-1050 (2003)

3. Kumar, A., Zhang, A.: Personal recognition using hand shape and texture. IEEE Transaction on Image Processing 15(8), 2454-2461 (2006)

4. Zhang, L., Zhang, D.: Characterization of Palmprints by Wavelet Signatures via Directional Context Modeling. IEEE Transaction on Systems, Man, and Cybernetics 34(3), 1335-1347 (2004)

5. Wang, X., Gong, H., Zhang, H.: Palmprint Identification using Boosting Local Binary Pattern. In: 18th International Conference on Pattern Recognition, vol. 3, pp. 503-506 (2006)

6. Zhang, D., Shu, W.: Two novel characteristics in palmprint verification: Datum point invariance and line feature matching. Pattern Recognition 32(4), 691-702 (1999)

7. Han, C.-C., Cheng, H.-L., Lin, C.-L., Fan, K.-C.: Personal authentication using palmprint features. Pattern Recognition 36, 371-381 (2003)

8. International Committee for Information Technology Standards. Technical Committee M1-Biometrics (2005), http: / / www. incits.org/tc_home/m1.htm

9. The PolyU palmprint database, http: / / www . comp.polyu.edu.hk/ biometrics

10. Wenxin, L., Zhang, D., Xu, Z.: Palmprint Identification by Fourier Transform. Intl. Journal of Pattern Recognition and Artificial Intelligence 16(4), 417-432 (2002)

11. Badrinath, G.S., Gupta, P.: An Efficient Multi-algorithmic Fusion System based on Palmprint for Personnel Identification. In: Intl. Conf. on Advanced Computing, pp. 759-764 (2007)

12. Ross, A., Jain, A.K.: Information fusion in biometrics. Pattern Recognition Letters, 21152125 (2003)

13. Pavlidis, T.: Algorithms for graphics and image processing. Springer, Heidelberg (1982)

14. Bay, H., Tuytelaars, T., Van Gool, L.: SURF: Speeded up robust features. In: Leonardis, A., Bischof, H., Pinz, A. (eds.) ECCV 2006. LNCS, vol. 3951, pp. 404-417. Springer, Heidelberg (2006)

15. Lu, G., Wang, K., Zhang, D.: Wavelet based independent component analysis for palmprint identification. In: Intl. conf. on Machine Learning and Cybernetics, pp. 3547-3550 (2004)

16. Wang, Y., Ruan, Q.: Kernel Fisher Discriminant Analysis for Palmprint Recognition. In: 18th Intl. Conf. on Pattern Recognition, pp. 457-460 (2006)

17. Bay, H., Fasel, B., Van, L.: Interactive museum guide: Fast and robust recognition of museum objects. In: First Intl. workshop on mobile vision (2006) 
18. Murillo, A.C., Guerrero, J.J., Sagues, C.: SURF features for efficient robot localization with omnidirectional images. In: IEEE Intl. Conf. on Robotics and Automation, pp. 39013907 (2007)

19. Viola, P., Jones, M.: Rapid object detection using a boosted cascade of simple features. In: IEEE Conf. on Computer Vision and Pattern Recognition, pp. 511-518 (2001)

20. Shu, W., Zhang, D.: Automated Personal Identification by Palmprint. Optical Engineering 37(8), 2359-2362 (1998)

21. Mikolajczyk, K., Schmid, C.: A performance evaluation of local descriptors. Pattern Analysis and Machine Intelligence 27, 1615-1630 (2005)

22. Independent Testing of Iris Recognition Technology Final Report. International Biometric Group (2005) 